\title{
Cross-Sectional Study on Vitamin D, Zinc Oxide and Fatty Acid Status in a Population with a Moderate to High Risk of AMD Identified by the STARS ${ }^{\circledR}$ Questionnaire
}

Julie Jacob • Els Mangelschots · Marine Michez • Serdal N. Sanak •

Anita Leys

Received: December 21, 2020 / Accepted: January 23, 2021 / Published online: February 23, 2021

(C) The Author(s) 2021

\section{ABSTRACT}

Introduction: A prospective study was carried out in Belgium to determine the proportion of subjects with a moderate to high risk of developing age-related macular degeneration (AMD), identified using the STARS ${ }^{\circledR}$ (Simplified Théa AMD Risk-Assessment Scale) questionnaire, who were in need of nutritional supplementation, by assessing the vitamin $\mathrm{D}$, zinc oxide and fatty acid profile status.

Methods: This multicentre cross-sectional pilot study involved 50 Belgian subjects with no or early AMD, aged $>55$ years who were at moderate to high risk for AMD. Subjects were

Supplementary Information The online version of this article (https://doi.org/10.1007/s40123-021-003354) contains supplementary material, which is available to authorized users.

J. Jacob $(\bowtie) \cdot$ A. Leys

Department of Ophthalmology, Universitair

Ziekenhuis Leuven (UZ Leuven), Leuven, Belgium

e-mail: Julie.jacob@uzleuven.be

E. Mangelschots

Oogartsenpraktijk Alken, Alken, Belgium

M. Michez

Cliniques Universitaires Saint Luc, Université catholique de Louvain (UCLouvain), Brussels, Belgium

S. N. Sanak

Department of Ophthalmology, CHIREC-Hôpital

Delta, Brussels, Belgium assessed using the STARS ${ }^{\circledR}$ questionnaire, visual acuity assessment, an optical coherence tomography scan of the macula and fundus photography. Blood samples were collected, and serum analyses were performed to determine the the omega-6:omega-3 $(\Omega 6: \Omega 3)$ ratio and the levels of eicosapentaenoic acid (EPA), docosahexaenoic acid (DHA), zinc and cupric oxides and vitamin D.

Results: Abnormal serum levels for at least one of the micronutrients was detected in $94 \%$ of the subjects. Lower than optimal vitamin D levels were found in $76 \%$ of the participants, and $68 \%$ of the subjects demonstrated at least one abnormal fatty acid profile. The $\Omega 6: \Omega 3$ ratio was above the reference range for normal values in 54\% of the subjects; DHA and EPA levels were below the reference range in 60 and $46 \%$ of the subjects, respectively; and zinc oxide concentration was below the reference range in $50 \%$ of the subjects. Only $12 \%$ of the subjects exhibited cupric oxide deficiency.

Conclusion: In this study, the STARS ${ }^{\circledR}$ questionnaire was used for early identification of patients at moderate to high risk of AMD in real life. These patients presented a suboptimal nutritional status. Further research is needed to determine if specific diet modification or micronutrient supplement intake delays the onset or slows down the progression of AMD in these subjects.

Trial Registration: Trial registration: ClinicalTrials.Gov, identifier: NCT04482465. 
Keywords: AMD; Micronutrients; Omega-6/ omega-3; Vitamin D; Zinc

\section{Key Summary Points}

This study evaluated the correlation between risk factors for age-related macular degeneration (AMD), identified using the validated STARS ${ }^{\circledR}$ questionnaire, with vitamin D status in a Belgium population.

Early identification of persons at risk for AMD is linked with better long-term outcome, emphasizing the need for a simple and fast tool to identify those at risk of AMD.

The results of this study confirm the robustness of the STARS ${ }^{\circledR}$ questionnaire for early identification of AMD and also confirm a low vitamin D status in persons at risk of AMD.

\section{DIGITAL FEATURES}

This article is published with digital features, including a summary slide to facilitate understanding of the article. To view digital features for this article go to https://doi.org/10.6084/ m9.figshare.13615448.

\section{INTRODUCTION}

Age-related macular degeneration (AMD), a degenerative disease of the macula that leads to progressive visual impairment, is the leading cause of permanent vision loss among adults more than 60 years old in the Western World [1]. The disease's early stage is characterized by drusen and pigmentary abnormalities, with possible progression to a late stage that is further categorized into two distinct subtypes: geographic atrophy or choroidal neovascularization, also known as dry or wet AMD, respectively [2]. AMD severity can be classified into four grades using the Age-Related Eye Disease Study (AREDS) simplified scale based on examination findings of drusen, atrophy and neovascularization: no AMD, early AMD, intermediate AMD and advanced AMD [3].

Oxidative stress, inflammation and angiogenesis in the retinal pigment epithelium (RPE) and choriocapillaries are key processes in AMD pathogenesis, progressively leading to photoreceptor loss [4]. The multifactorial pathogenesis of AMD results from the interaction of environmental, genetic, metabolic, diet and lifestyle factors. Numerous epidemiological studies have investigated associations of various risk factors with the development and progression of AMD. A meta-analysis of 24 studies demonstrated that age, smoking and family history of AMD are the risk factors that show the strongest and most consistent associations with AMD, with moderate associations reported for body mass index (BMI), history of hypertension and history of cardiovascular disease [5]. Other proposed risk factors for AMD include gender, ethnicity, diabetes, hypercholesteraemia, iris colour [5], history of atherosclerosis [6], refractory errors $[7,8]$, diet and nutrition $[9,10]$.

The multifactorial epidemiology of AMD is still a subject of investigation. Identifying the AMD risk of an individual in clinical practice is difficult. Building on that difficulty, the Simplified AMD Risk-Assessment Scale (STARS ${ }^{\circledR}$ ) [11] questionnaire was developed as a scoring system to easily and rapidly screen people with moderate-to-high risk for AMD in routine clinical practice. The STARS ${ }^{\circledR}$ questionnaire comprises 13 items covering family history of AMD, demographic, medical history and ocular risk factors (Table 1; Electronic Supplementary Material Fig. 1). It is self-administered and enables ophthalmologists to assess the personalized risk for AMD, with the purpose of delivering appropriate early clinical management in eye-care practice [11]. The STARS ${ }^{\circledR}$ questionnaire was validated in two large patient samples, one sample of 12,639 patients in Italy and one sample of 6837 patients in France. The score showed good discrimination in both samples (area under the receiver operating characteristic [ROC] curve of 0.78 and 0.72 in the Italian and French samples, respectively) [11]. 
Table 1 Demographic characteristics of the participants in the study

\begin{tabular}{|c|c|c|}
\hline & $\begin{array}{l}\text { Subjects }(n / \\
50)\end{array}$ & $\begin{array}{l}\text { Subjects } \\
(\%)\end{array}$ \\
\hline \multicolumn{3}{|l|}{ Sex } \\
\hline Male & 21 & 42 \\
\hline Female & 29 & 58 \\
\hline \multicolumn{3}{|l|}{ Age } \\
\hline Age $55-65$ & 20 & 40 \\
\hline $65-75$ & 22 & 44 \\
\hline $75-85$ & 6 & 12 \\
\hline 85 & 2 & 4 \\
\hline \multicolumn{3}{|l|}{ Ethnicity } \\
\hline Caucasian/Asian & 49 & 98 \\
\hline Subsaharan African & 0 & 0 \\
\hline $\begin{array}{l}\text { North African/Middle } \\
\text { east }\end{array}$ & 1 & 2 \\
\hline \multicolumn{3}{|l|}{ AMD family history } \\
\hline No & 30 & 60 \\
\hline Yes & 20 & 40 \\
\hline \multicolumn{3}{|l|}{ Smoking history } \\
\hline Never smoked & 28 & 56 \\
\hline $\begin{array}{l}\text { Current or former } \\
\text { smoker }\end{array}$ & 22 & 44 \\
\hline \multicolumn{3}{|l|}{ Body Mass Index (BMI) } \\
\hline $\mathrm{BMI}<25$ & 24 & 48 \\
\hline BMI 25-30 & 19 & 38 \\
\hline $\mathrm{BMI}>30$ & 7 & 14 \\
\hline \multicolumn{3}{|c|}{ History of myocardial infarction (MI) } \\
\hline No & 47 & 94 \\
\hline Yes & 2 & 4 \\
\hline Missing data & 1 & 2 \\
\hline \multicolumn{3}{|c|}{ History of hypertension (HT) } \\
\hline No & 32 & 64 \\
\hline Yes & 18 & 36 \\
\hline History of atherosclerosis & thScl) & \\
\hline
\end{tabular}

Table 1 continued

\begin{tabular}{lll}
\hline & $\begin{array}{l}\text { Subjects }(\boldsymbol{n} / \\
\mathbf{5 0})\end{array}$ & $\begin{array}{l}\text { Subjects } \\
(\%)\end{array}$ \\
\hline No & 41 & 82 \\
Yes & 8 & 16 \\
Missing data & 1 & 2 \\
History of hypercholesterolemia (Hyperchol) & \\
No & 23 & 46 \\
Yes & 27 & 54 \\
Cataract surgery & & \\
No & 34 & 68 \\
Yes & 16 & 32 \\
Refractive errors & & \\
$\geq 1$ Myopia & 19 & 38 \\
$\geq 1$ Hypermetropia & 18 & 36 \\
No errors & 13 & 26 \\
Risk for AMD & & 98 \\
Moderate (10-19) & 49 & 2 \\
High ( $\geq 20)$ & 1 & \\
\hline
\end{tabular}

The 13 characteristics presented are the age-related macular degeneration (AMD) risk factors identified in the Simplified Théa AMD Risk-Assessment Scale (STARS ${ }^{\circledR}$ ) questionnaire

Establishing a risk prediction algorithm is equally important to the early identification of modifiable risk factors for AMD, allowing for the appropriate interventions to modify the risk for development or progression of the disease. Some lifestyle modifications linked to associated risk factors include smoking cessation [12] and diet modification to lower the BMI, decrease the risk of cardiovascular disease and increase micronutrient intake [9]. The protective effect of such modifications on AMD development and progression has been clinically demonstrated for several micronutrients with anti-inflammatory and/or anti-oxidant 
and/or anti-angiogenic properties in the AREDS [3] and other studies [3, 10, 13-16]. These include carotenoids (lutein and zeaxanthin, $\beta$ carotene), vitamins $\mathrm{A}, \mathrm{C}, \mathrm{E}, \mathrm{D}$, minerals (zinc oxide, cupric oxide) and dietary polyunsaturated fatty acids (PUFAs).

Serum analysis for measuring the levels of micronutrients that are proposed to contribute to decreasing the risk for AMD is expensive and not performed as part of routine practice in ophthalmological clinics. We have conducted this prospective study in Belgium to estimate the proportion of subjects at moderate to high risk of AMD based on the STARS ${ }^{\circledR}$ questionnaire. The serums of those identified to be at risk for AMD were then assessed for vitamin $\mathrm{D}$, zinc oxide, cupric oxide and fatty acid levels to evaluate the prevalence of suboptimal nutritional status. This investigation may provide evidence indicating the need for patients predicted to be at risk of developing AMD, as assessed by the STARS $^{\circledR}$ questionnaire, to increase or adjust relevant micronutrient intake.

\section{METHODS}

\section{Study Population}

This pilot study is a multicentre cross-sectional study of an exploratory nature, hence no power calculation was performed; the aim was to demonstrate trends. The study includes 50 subjects without a control group.

Fifty people were enrolled in the study. The inclusion criteria were: age $\geq 55$ years with a STARS $^{\circledR}$ score $\geq 10$ (moderate-to-high risk for AMD). Individuals were excluded from the study if they had a STARS $^{\circledR}$ score $<10$, were already taking nutritional supplements containing vitamin $\mathrm{D}$ or trace nutrients in the 4 months prior to enrolment or had grade- 3 or grade-4 AMD scores according to the AREDS simplified scale. Patients were recruited in two centres between April and June 2017, namely the Alken Eye Clinic (25 patients) and the Ophthalmology Department of the University Hospitals Leuven (25 patients). Patients consulting for routine eye care or individuals accompanying patients were invited to participate in the study.

\section{Study Design and Procedures}

The study was conducted in accordance with the principles of Good Clinical Practice (GCP) and the Helsinki Declaration of 1964 and its later amendments. The study protocol and all relevant study documents were submitted for review and approved by the Ethics Committee Research UZ/KU Leuven (Ref. number: S59711). Informed consent was obtained from the subjects prior to their enrolment in the study and before the start of any study related procedures. All information collected was kept confidential and anonymized and used exclusively for the purposes of this study in compliance with the applicable personal data protection and processing laws.

The study was completed within a single visit. Outcome data were collected during a onetime subject interview comprising clinical eye examinations (typically visual acuity), the STARS $^{\circledR}$ questionnaire, visual acuity assessment, an optical coherence tomography scan of the macula and fundus photography. Blood samples were collected using a Vacutainer system, with one purple ethylenediaminetetraacetic acid (EDTA) tube and two red tubes to assay for the omega-3 fatty acids eicosapentaenoic acid (EPA) and docosahexaenoic acid (DHA) and omega- 6 fatty acids for the calculation of the omega-6:omega-3 ( $\Omega 6: \Omega 3)$ ratio, vitamin $\mathrm{D}$ [25(OH)D], zinc oxide and cupric oxide. Samples were analysed by the RP Lab, Mont-SaintGuibert, Belgium (http://www.rplab.be). The fatty acid profile was assessed using gas chromatography with the use of a flame ionization detector after an esterification process. Vitamin $25(\mathrm{OH}) \mathrm{D}$ was determined by competitive immunoassay testing, and zinc and cupric oxides were determined using atomic absorption spectroscopy.

\section{Data Analysis}

Data were tested for normality using the chisquared goodness-of-fit test. Data distribution 
was not normal for some of the assayed micronutrients, thus all data were analysed to identify trends using subject proportions (\%) and descriptive statistics for not normally distributed data (median and interquartile range [IQR, 25-75\%]). There are no missing data for the micronutrients analysed.

\section{Vitamin D Definition}

The definition of optimal vitamin D levels is key in shaping the recommendation for vitamin D intake. However, the guidelines for optimal vitamin D serum levels lack consistency between different organizations. The Institute of Medicine (IOM) [17] and Global Consensus [18] recommendations set the limit for optimal vitamin D levels at $20 \mu \mathrm{g} / \mathrm{L}$. The Endocrine Society recommendations set the minimal target value for optimal vitamin D levels at $30 \mu \mathrm{g} /$ $\mathrm{L}$, and the values of $<30$ and $<20 \mu \mathrm{g} / \mathrm{L}$ were labeled as insufficient or deficient, respectively [19]. The study populations on which these different recommendations are based vary. However, the majority of patients with AMD tend to be older and are, therefore, by default at higher risk for vitamin D deficiency; as such, they resemble more the population of the Endocrine Society. In the present study we have used the ranges recommended by the Endocrine Society.

\section{RESULTS}

\section{Subject Characteristics}

A total of 50 subjects were enrolled in this study. The demographic characteristics of these subjects are presented in Table 1 . At University Hospitals Leuven, 102 subjects were screened, and 25 were ultimately assessed to be eligible to participate in the study. Of those excluded from the study, 55 had a STARS ${ }^{\circledR}$ score $<10,17$ had a history of nutritional supplement intake in the 4 months prior to enrolment and five were classified with AMD grade 3 or 4 upon clinical examination. At the Alken Eye Clinic, 51 subjects were screened, of whom 25 were assessed to be eligible for participation in the study. Of those excluded, ten had a STARS ${ }^{\circledR}$ score $<10,13$ had a history of nutritional supplement intake in the 4 months prior to enrolment and three were classified with AMD grade 3 or 4 on the clinical examination.

\section{Micronutrient Levels}

Of the 50 subjects included in this study, 47 (94\%) had abnormal serum levels for at least one of the micronutrients analysed. The percentage of patients with abnormal results for each micronutrient analysed is presented in Fig. 1a-c.

\section{Vitamin D}

The median serum concentration of vitamin $25(\mathrm{OH}) \mathrm{D}$ in the study population was 23.9 (IQR 15.3-29.8) $\mu \mathrm{g} / \mathrm{L}$ (Fig. 1b). Of the 50 participants, lower than optimal vitamin D levels $(<30 \mu \mathrm{g} / \mathrm{L})$ [19] was evidenced in 38 (76\%), with 40 and $36 \%$ exhibiting vitamin D deficiency $(<20 \mu \mathrm{g} /$ L) [19] and vitamin D insufficiency (20-30 $\mu \mathrm{g} / \mathrm{L})$ [19], respectively (Fig. 1b, c). Most of the remaining participants $(92 \%, n=11 / 12)$ had borderline optimal values. This is reflected by a median concentration of 31.3 (IQR 30.6-37.7) $\mu \mathrm{g} / \mathrm{L}$ vitamin $\mathrm{D}$ for the subjects who had levels of vitamin D within the optimal range (Table 2). The median concentration of vitamin $\mathrm{D}$ among subjects who exhibited lower than optimal values was within the range of deficiency, estimated at 19.0 (IQR 13.9-26.5) $\mu \mathrm{g} / \mathrm{L}$ (Table 2).

Subgroup analysis for vitamin D median concentration suggested a trend for certain groups to exhibit a median concentration of vitamin $\mathrm{D}$ marginally below the deficiency threshold of $20 \mu \mathrm{g} / \mathrm{L}$. These groups and their corresponding median concentration of vitamin D were: males (18.4 [IQR 12.9-27.8] $\mu \mathrm{g} / \mathrm{L})$; overweight (18.4 [13.4-27.1] $\mu \mathrm{g} / \mathrm{L})$ and obese (17.7 [11.1-24.3] $\mu \mathrm{g} / \mathrm{L})$ and subjects with a history of hypertension (18.9 [1IQR 4.8-29.0] $\mu \mathrm{g} /$ L), atherosclerosis (17.7 [15.6-27.8] $\mu \mathrm{g} / \mathrm{L})$ and cataract surgery $(19.0[15.5-28.3] \mu \mathrm{g} / \mathrm{L})$. The vitamin $\mathrm{D}$ concentration of the only subject identified as being at high risk of AMD was $12.9 \mu \mathrm{g} / \mathrm{L}$ (Fig. 2). 


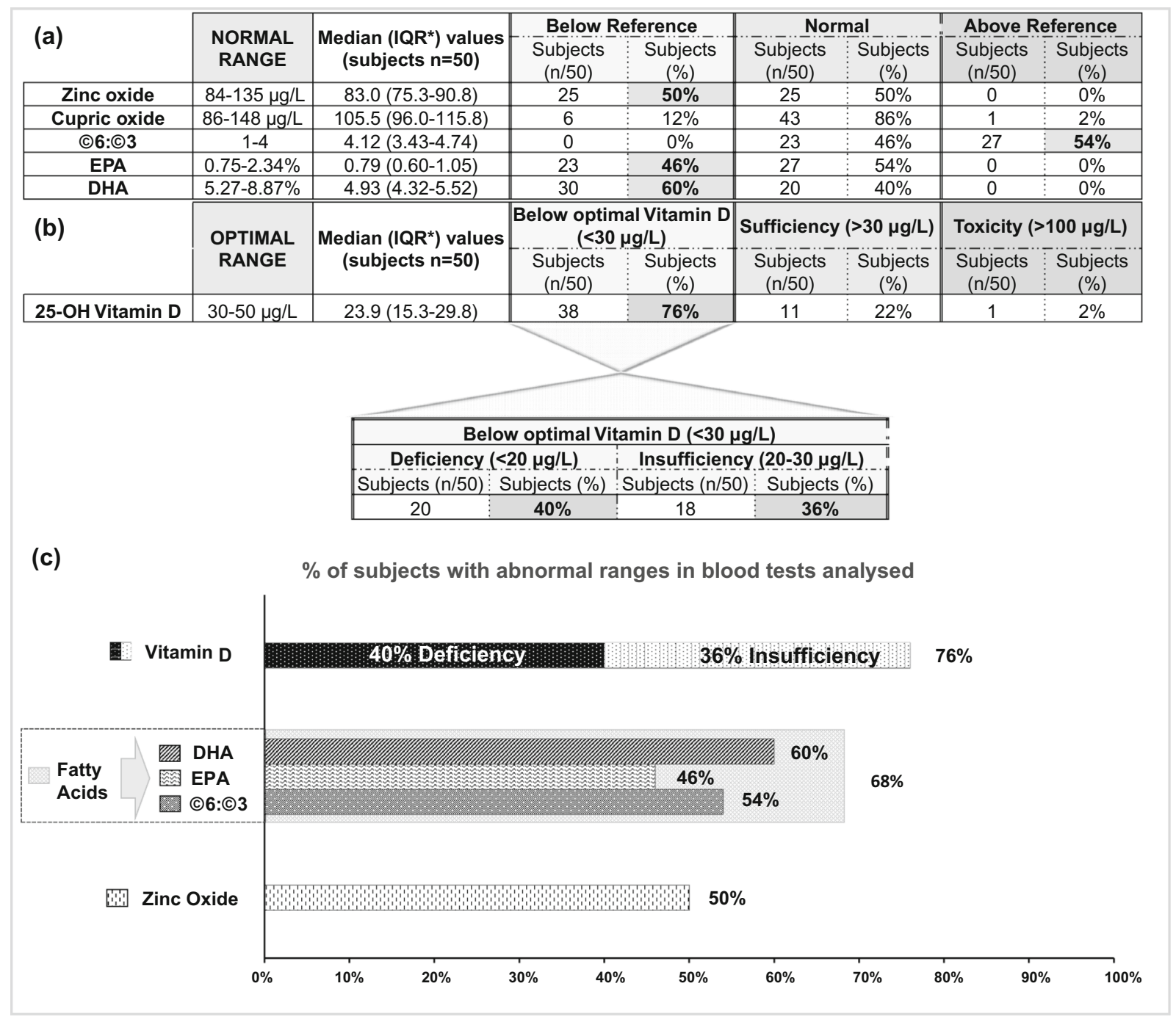

Fig. 1 a Number $(n)$ and proportion (\%) of subjects presenting with levels of zinc oxide, cupric oxide, eicosapentaenoic acid $(E P A)$ and docosahexaenoic acid $(D H A)$ below the range of reference values and with an omega-6:omega-3 ( $\Omega 6: \Omega 3)$ ratio above the range of reference values. Asterisk indicates interquartile range $(25-75 \%)$. b Number $(n)$ and proportion (\%) of subjects

\section{Fatty Acid Levels}

Of the 50 subjects, $68 \%$ had at least one abnormal fatty acid $(\Omega 6: \Omega 3$ ratio, EPA or DHA) profile, (Fig. 1c). The $\Omega 6: \Omega 3$ ratio was above the reference range (normal range of $\Omega 6: \Omega 31-4$ ) in $54 \%$ of the subjects, and the median $\Omega 6: \Omega 3$ ratio in the study population was 4.12 (IQR 3.43-4.74) (Fig. $1 \mathrm{a}, \mathrm{c}$ ). The median $\Omega 6: \Omega 3$ ratio presenting with vitamin $\mathrm{D}$ levels below the optimal range. These subjects were further analysed to identify the number and proportion of them exhibiting vitamin $\mathrm{D}$ insufficiency or vitamin D deficiency (36 and 40\%, respectively). c Graph representation of the percentage of subjects with abnormal serum levels of micronutrients tested

among subjects who had values above the reference range was 4.71 (IQR 4.35-5.10) (Table 2). DHA levels were below reference range (normal range of DHA $5.27-8.87 \%$ ) in $60 \%$ of the subjects, with the median concentration in the study population being $4.93 \%$ (IQR 4.32-5.52\%) (Fig. 1a, c). The median DHA concentration among subjects with levels below the 
Table 2 Serum concentrations of micronutrients in the study population

\begin{tabular}{|c|c|c|c|c|c|}
\hline & $\begin{array}{l}\text { Normal } \\
\text { range }\end{array}$ & $\begin{array}{l}\text { Total } \\
\text { population }\end{array}$ & $\begin{array}{l}\text { Population below } \\
\text { reference }\end{array}$ & $\begin{array}{l}\text { Population within } \\
\text { normal range }\end{array}$ & $\begin{array}{l}\text { Population above } \\
\text { reference }\end{array}$ \\
\hline Zinc oxide & $\begin{array}{c}84-135 \\
\mu \mathrm{g} / \mathrm{L}\end{array}$ & $83.0(75.3-90.8)$ & $75.0(72.0-78.0)$ & $91.0(86.0-99.0)$ & $\mathrm{N} / \mathrm{A}^{* *}$ \\
\hline Cupric oxide & $\begin{array}{c}86-148 \\
\mu \mathrm{g} / \mathrm{L}\end{array}$ & $105.5(96.0-115.8)$ & $81.5(80.0-83.0)$ & $108.0(98.0-116.5)$ & $\mathrm{N} / \mathrm{A}^{* *}$ \\
\hline$\Omega 6: \Omega 3$ & $1-4$ & $4.12(3.43-4.74)$ & $\mathrm{N} / \mathrm{A}^{* *}$ & $3.41(3.23-3.77)$ & $4.71(4.35-5.10)$ \\
\hline $\mathrm{EPA}$ & $0.75-2.34 \%$ & $0.79(0.60-1.05)$ & $0.58(0.48-0.67)$ & $1.04(0.85-1.13)$ & $\mathrm{N} / \mathrm{A}^{* *}$ \\
\hline DHA & $5.27-8.87 \%$ & $4.93(4.32-5.52)$ & $4.48(3.99-4.74)$ & $5.58(5.28-5.79)$ & $\mathrm{N} / \mathrm{A}^{* *}$ \\
\hline $\begin{array}{l}25-\mathrm{OH} \\
\text { vitamin D }\end{array}$ & $\begin{array}{r}30-50 \\
\mu \mathrm{g} / \mathrm{L}\end{array}$ & $23.9(15.3-29.8)$ & $19.0(13.9-26.5)$ & $31.3(30.6-37.7)$ & $\mathrm{N} / \mathrm{A}^{* *}$ \\
\hline
\end{tabular}

Values are presented as the median concentration and IQR (25-75\%, as a measure of variability) of all nutrients analysed for subjects who presented with values below the reference range, within the normal range and above the reference range $E P A$ Eicosapentaenoic acid, DHA docosahexaenoic acid, $I Q R$ interquartile range (25-75\%), N/A not applicable, $\Omega 6: \Omega 3$ omega-6:omega-3 ratio

The bold values are median concentration of vitamin $\mathrm{D}$ in the subjects presenting with values below optimal was in the spectrum of vitamin D deficiency $(<20 \mu \mathrm{g} / \mathrm{L})$ and median concentration in those subjects within optimal vitamin D range $(30-50 \mu \mathrm{g} / \mathrm{L})$ was borderline within range

reference range was $4.48 \%$ (IQR $3.99-4.74 \%$ ) (Table 2). EPA levels were lower than the reference range (normal range of EPA $0.75-2.34 \%$ ) in $46 \%$ of the subjects, with the median concentration in the study population being 0.79 (IQR 0.60-1.05) \% (Fig. 1a, c). The median EPA concentration among subjects with levels below the reference range was $0.58 \% \quad(0.48-0.67 \%)$ (Table 2).

\section{Zinc Oxide and Cupric Oxide}

Zinc oxide concentration was found to be below the reference range (normal range of zinc oxide $84-135 \mu \mathrm{g} / \mathrm{L}$ ) in $50 \%$ of the subjects, and the median concentration in the study population was 83.0 (IQR 75.3-90.8) $\mu \mathrm{g} / \mathrm{L}$ (Fig. 1a, c). In those subjects whose median zinc oxide concentration was lower than the reference values, the median zinc oxide level was 75.0 (IQR 72.0-78.0) $\mu \mathrm{g} / \mathrm{L}$ (Table 2). Cupric oxide levels were within the normal range $(86-148 \mu \mathrm{g} / \mathrm{L})$ for the majority of the tested population (86\%), with the median concentration in the study population being 105.5 (IQR 96.0-115.8) $\mu \mathrm{g} / \mathrm{L}$
(Fig. 1a). In those subjects with cupric oxide levels below reference range (12\%), the median cupric oxide concentration was 81.5 (IQR 80.0-83.0) $\mu \mathrm{g} / \mathrm{L}$ (Table 2).

\section{DISCUSSION}

In this study we used the STARS ${ }^{\circledR}$ questionnaire in a Belgian population aged $\geq 55$ years who had either no AMD or early AMD to identify people at moderate-to-high risk for developing AMD or AMD progression. The STARS ${ }^{\circledR}$ questionnaire has been compiled from validated risk factors for AMD and can be used to screen and identify healthy or early-stage AMD patients, such as the subjects of this study, who are at risk of AMD development or progression. Early detection of those individuals is of utmost importance as it will enable ophthalmologists to put in place an early strategy of prevention $[11,20]$.

Blood serum samples of the subjects identified to be at risk of AMD were analysed for 


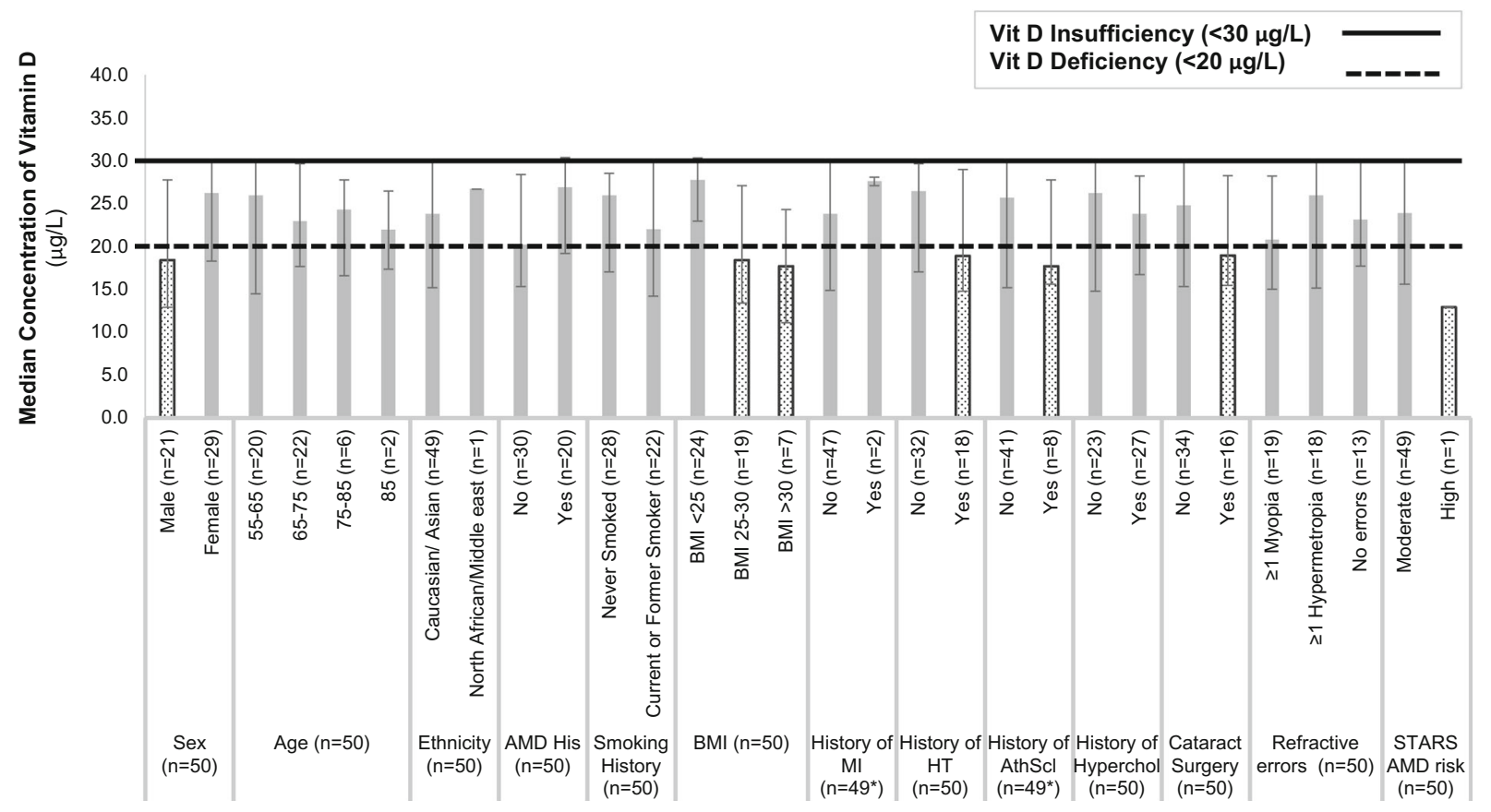

Fig. 2 Subgroup analysis for vitamin D median concentration depicting a trend for certain groups. Dotted boxes (male, subjects with BMI $>25$, subjects with a history of hypertension, atherosclerosis and cataract surgery, subjects at high AMD risk) represent groups exhibiting a median concentration of vitamin $\mathrm{D}$ below the vitamin $\mathrm{D}$

vitamin $\mathrm{D}$, fatty acids, zinc oxide and cupric oxide levels, with the results showing that the vast majority (94\%) of the subjects presented abnormal levels of at least one of these micronutrients.

Poor diet and lack of micronutrients with anti-oxidant, anti-inflammatory and anti-angiogenic properties are considered to be risk factors for AMD $[9,11]$. The beneficial effect of adopting specific diet patterns rich in nutrients associated with a reduced risk for AMD has been previously demonstrated [21, 22]. However, identification of the appropriate beneficial diet pattern and adherence to it pose issues that render the importance of micronutrient supplement intake unequivocal. The importance for additional intake of micronutrient supplements in AMD is especially highlighted in the elderly who are at higher risk of micronutrient deficiency and show low adherence to specific diet patterns. It has been demonstrated by Koning et al. that only $3.7 \%$ of elderly patients deficiency threshold of $20 \mu \mathrm{g} / \mathrm{L}$. Grey shaded boxes represent groups exhibiting a median concentration of vitamin $\mathrm{D}$ between deficiency $(20 \mu \mathrm{g} / \mathrm{L})$ and insufficiency levels $(30 \mu \mathrm{g} / \mathrm{L})$. Asterisk indicates that data were not available for 1 out of 50 subjects

followed the recommended nutrient intake [21].

The benefit of micronutritional diet supplements for AMD has been established by numerous studies [3, 13, 23]. Micronutrients that make up the currently existing AREDS proposed formulation of dietary supplements are lutein/zeaxanthin, $\beta$-carotene (contraindicated for smokers) [24], vitamin C, vitamin E, zinc oxide and cupric oxide $[3,23]$. There is increasing interest in boosting the synergistic action of supplements already being used by adding other anti-oxidant micronutrients and components with plausible biological effect, such as omega-3 PUFAs [25], resveratrol [26], and vitamin D [4].

\section{Zinc Oxide}

The concentration of zinc in the serum has been found to be significantly lower in AMD patients 
than in healthy subjects [27]. In our study, only half of the subjects in this study exhibited normal levels of zinc oxide, potentially indicating the need to increase their intake. The protective effect of zinc supplementation for early and late AMD is supported by numerous studies [3, 13, 25, 28]. Zinc has key anti-oxidant and anti-apoptotic properties and is abundant in the RPE and photoreceptor layers. Zinc levels decrease in the retina due to aging, possibly contributing to AMD development by causing impaired cellular metal homeostasis, thereby inducing RPE and photoreceptor degradation and promoting oxidative stress [29]. The daily zinc supplement of $80 \mathrm{mg}$ recommended by AREDS 2001 [3] is much higher than the recommended upper tolerance level for zinc intake [30]. This recommendation was reduced to $25 \mu \mathrm{g}$ in AREDS2 (2013) [13], and the Blue Mountains Eye Study indicated a threshold dosage of $15.8 \mathrm{mg}$ to avoid any side effect [28].

\section{Fatty Acids}

Patients with AMD have been previously reported to have significantly lower levels of DHA and EPA than healthy subjects [31]. Abnormal levels of at least one fatty acid profile was found in $68 \%$ of our study subjects. DHA and EPA insufficiency was observed in 60 and $46 \%$ of the subjects, respectively. DHA and EPA are abundantly found in the retina and their anti-oxidative, anti-apoptotic and anti-inflammatory effects are of particular interest in AMD. PUFAs may increase the retinal density of the macular pigment which has local anti-oxidant and anti-inflammatory function. A disruption of their balance in the retina leads to photoreceptor degradation and an accumulation of lipid and lipoprotein debris in the RPE layer [32]. Given that continued regeneration of the retinal membrane requires constant supply of omega-3 fatty acids from RPE cells, an EPA/ DHA-rich diet, such as fish, should improve retinal function and slow the development of AMD, as demonstrated in the Blue Mountains Eye Study for wet AMD [33]. Several longitudinal studies have also demonstrated associations of decreased AMD risk for early or late AMD development with the highest consumption of omega-3 PUFAs, some just for DHA [14, 34, 35] and others for both DHA and EPA $[25,36]$.

Low levels of omega- 3 fatty acids may have clinical implications in conjunction with high levels of omega- 6 fatty acids leading to a higher than normal ratio of $\Omega 6: \Omega 3$, which was found in $54 \%$ of our subjects. A high ratio of omega6:omega-3 ratio and a high dietary intake of the monounsaturated fatty acid omega- 6 , known for its pro-inflammatory and pro-angiogenic properties, have been previously to be associated with an increased risk for AMD [37]. In addition, one study found that AMD risk reduction due to omega 3 intake occured mainly in individuals with low levels of omega 6 intake [38].

\section{Vitamin D}

Our data analysis revealed an overwhelming majority $(76 \%)$ of the subjects to be insufficient or deficient in vitamin D. Strikingly, the remaining subjects with levels within the optimal range had borderline values despite having been screened in the summer when vitamin D concentrations are the highest. Over 50\% of the world's population is at risk of vitamin D deficiency [39]. The elderly in particular are exposed to dietary vitamin $\mathrm{D}$ deficit, reduced mobility leading to reduced exposure to sunlight and renal function impairment-all factors that converge to vitamin D deficiency [40].

In support of our results, it has been previously reported that $87.3 \%$ of patients with AMD exhibited low levels of vitamin D intake, which was correlated with a higher risk of AMD progression [16]. The association of vitamin D shortage [assessed based on serum 25(OH)D] with early or late-type AMD has been reported in several cross-sectional studies, but remains under debate, as demonstrated in subsequent meta-analyses. $\mathrm{Wu}$ et al. concluded that there was no consistent evidence proving an inverse association between serum vitamin D levels and risk for AMD [41], in agreement with a recent meta-analysis by Ferreira et al., although the latter demonstrated a trend for late AMD among patients with vitamin D deficiency [42]. On the 
contrary, Annweiler et al. concluded in their meta-analysis that vitamin D dietary intake has a protective effect against AMD [15]. The latter view is supported by the results of a longitudinal prospective study performed by Merle et al. [16]. The association between vitamin D intake and AMD requires further assessment in prospective randomized controlled trials, as cross-sectional studies are not designed to determine causality. Recent data from the first randomized clinical trial evaluating vitamin $D$ in AMD prevention did not exhibit a significant effect of vitamin D supplementation on AMD onset or progression [43]. Several limitations of this study, which was predominantly designed to evaluate vitamin D intake for the prevention of cancer and cardiovascular diseases, include the well-nourished study population, the lack of stratification by clinical manifestation of AMD, the small number of events per subgroup, the short follow-up, the self-reported AMD diagnosis, the lack of adjustment for multiple comparisons and reduced statistical power. These limitations should be taken into account in the design of future randomized trials that aim to further elucidate the potential effect of vitamin $D$ intake on AMD risk in the general population and distinct clinical subgroups of AMD patients.

The fact remains that both experimental in vitro and in vivo evidence support a protective role of vitamin D in AMD through the inhibition of oxidation, inflammation and angiogenesis and fibrosis pathogenic processes in the RPE and choroid [4, 44]. Consistently, of particular interest is the observation that vitamin D insufficiency is associated with an increased likelihood to develop subretinal fibrosis (SF) in patients with neovascular AMD. Preventing subretinal fibrosis is of vital importance to preclude subsequent irreversible retinal damage and subsequent vision loss, as in contrast to neovascularization, SF does not respond well to treatment and becomes steadily more prominent $[45,46]$. Thus, it would be interesting to further assess the effect of vitamin D supplementation on AMD in large population randomized trials, analysed in a stratified by clinical manifestation of AMD manner, including neovascular AMD with or without SF.
Vitamin D multifunctional bioactivity should not be overlooked when considering the potential clinical significance of its intake in AMD. Vitamin D deficiency has also been linked to conditions associated with AMD risk, including obesity [47], myocardial infarction [48], hypertension and coronary disease [49] and cataract [50]. It is conceivable that this is reflected in the trend for deficiency $(<20 \mu \mathrm{g} / \mathrm{L})$ we observed for overweight and obese subjects, and subjects with a history of hypertension, atherosclerosis, myocardial infarction and cataract surgery; however, these trends were marginal in our study, and their significance should be tested in larger populations. Consequently, the recommendation for vitamin $\mathrm{D}$ intake to AMD patients may prove beneficial in a cumulative manner.

The clinical implication of this study is that ophthalmologists may use the STARS ${ }^{\circledR}$ questionnaire to identify patients at risk for AMD and offer advice for a diet rich in relevant nutrients, as well as recommend, when necessary, micronutrient supplements. The potential limitations of the study arise from the small sample size of the study and the lack of a control group. The study was not powered to test the statistical significance of the detected abnormalities, to evaluate the extent of micronutrient deficiency for different levels of AMD risk or to detect all of the potential associations of abnormal nutrient levels with specific AMD risk factors. In addition, only a small number of nutrients was included, providing a very crude assessment of the overall nutritional status for these patients. It would be interesting to assess a more extensive nutritional profile in a follow-up study. Future investigations addressing all of these issues in a large population could potentially provide insights for a personalized and comprehensive nutrient supplementation plan.

\section{CONCLUSIONS}

Assays for serum micronutrient values are expensive and not routinely performed in clinical practice by ophthalmologists. It is perfectly rational to advocate a healthy diet and daily 
exposure to sunshine and outdoor light with the aim to optimize the nutritional status of people at risk for AMD. Many studies have shown the potential benefit of micronutrient supplementation against AMD progression. Our data collectively suggest that Belgians at moderate-to-high risk of AMD development or progression, identified using the inexpensive STARS $^{\circledR}$ questionnaire, present with a suboptimal nutritional status. Increasing the intake of omega 3 fatty acids, vitamin D and zinc could potentially delay the onset or slow down the progression of this condition but this needs to be confirmed by further research, particularly large randomized trials assessing the association of vitamin D intake and distinct clinical manifestations of AMD.

\section{ACKNOWLEDGEMENTS}

The authors would like to thank Dr Van Keer, Dr Breda, Dr Vanderschueren and Kimberly Motmans for their contribution in the recruitment of the study population. We also thank the participants of the study.

Funding. This study was funded by a grant from Laboratoires Théa, 12 rue Louis-Blériot, 63000 Clermont-Ferrand. The study sponsor also funded the journal's Rapid Service Fee.

Medical Writing. The authors would like to thank Dr Maria Notaridou who provided medical writing services on behalf of Creative Pharma Services S.M.S.A.

Authorship. All named authors meet the International Committee of Medical Journal Editors (ICMJE) criteria for authorship for this article, take responsibility for the integrity of the work as a whole, and have given their approval for this version to be published.

Disclosures. Julie Jacob, Els Mangelschots, Marine Michez, Serdal N. Sanak, Anita Leys declare that there is nothing to disclose.

Compliance with Ethics Guidelines. The study was conducted in accordance with the principles of Good Clinical Practice (GCP) and Helsinki Declaration of 1964 and its later amendments. The study protocol and all relevant study documents were submitted for review and approved by the Ethics Committee Research UZ/KU Leuven (Ref. number: S59711). Informed consent was obtained from the subjects prior to their enrolment in the study and before the start of any study related procedures.

Data Availability. The datasets generated during and/or analysed during the current study are available from the corresponding author on reasonable request.

Open Access. This article is licensed under a Creative Commons Attribution-NonCommercial 4.0 International License, which permits any non-commercial use, sharing, adaptation, distribution and reproduction in any medium or format, as long as you give appropriate credit to the original author(s) and the source, provide a link to the Creative Commons licence, and indicate if changes were made. The images or other third party material in this article are included in the article's Creative Commons licence, unless indicated otherwise in a credit line to the material. If material is not included in the article's Creative Commons licence and your intended use is not permitted by statutory regulation or exceeds the permitted use, you will need to obtain permission directly from the copyright holder. To view a copy of this licence, visit http://creativecommons.org/licenses/by$\mathrm{nc} / 4.0 /$.

\section{REFERENCES}

1. Wong WL, et al. Global prevalence of age-related macular degeneration and disease burden projection for 2020 and 2040: a systematic review and meta-analysis. Lancet Glob Health. 2014;2(2): e106-16.

2. Lim LS, et al. Age-related macular degeneration. Lancet. 2012;379(9827):1728-38.

3. Group A-REDSR. A randomized, placebo-controlled, clinical trial of high-dose supplementation with vitamins $\mathrm{C}$ and $\mathrm{E}$, beta carotene, and zinc for age- 
related macular degeneration and vision loss: AREDS report no. 8. Arch Ophthalmol. 2001;119(10):1417-36.

4. Layana AG, et al. Vitamin D and age-related macular degeneration. Nutrients 2017;9:1120. https:// doi.org/10.3390/nu9101120.

5. Chakravarthy U, et al. Clinical risk factors for agerelated macular degeneration: a systematic review and meta-analysis. BMC Ophthalmol. 2010;10:31.

6. Vingerling JR, et al. Age-related macular degeneration is associated with atherosclerosis: the Rotterdam Study. Am J Epidemiol. 1995;142(4):404-9.

7. Pan CW, et al. Refractive errors and age-related macular degeneration: a systematic review and meta-analysis. Ophthalmology. 2013;120(10): 2058-65.

8. Li Y, et al. Refractive error and risk of early or late age-related macular degeneration: a systematic review and meta-analysis. PLoS One. 2014;9(3): e90897.

9. Chapman NA, Jacobs RJ, Braakhuis AJ. Role of diet and food intake in age-related macular degeneration: a systematic review. Clin Exp Ophthalmol. 2019;47(1):106-27.

10. Heesterbeek TJ, et al. Risk factors for progression of age-related macular degeneration. Ophthalmic Physiol Opt. 2020;40(2):140-70.

11. Delcourt C, et al. Development and validation of a risk score for age-related macular degeneration: the STARS Questionnaire. Investig Ophthalmol Vis Sci. 2017;58(14):6399-407.

12. Velilla $\mathrm{S}$, et al. Smoking and age-related macular degeneration: review and update. J Ophthalmol. 2013;2013:895147.

13. Chew EY, et al. Long-term effects of vitamins $\mathrm{C}$ and $\mathrm{E}, \quad \beta$-carotene, and zinc on age-related macular degeneration: AREDS report no. 35. Ophthalmology. 2013;120(8):1604-2114.

14. Reynolds R, Rosner B, Seddon JM. Dietary omega-3 fatty acids, other fat intake, genetic susceptibility, and progression to incident geographic atrophy. Ophthalmology. 2013;120(5):1020-8.

15. Annweiler C, et al. Circulating vitamin D concentration and age-related macular degeneration: systematic review and meta-analysis. Maturitas. 2016;88:101-12.

16. Merle BMJ, et al. Associations between vitamin D intake and progression to incident advanced age- related macular degeneration. Invest Ophthalmol Vis Sci. 2017;58(11):4569-78.

17. Ross AC, et al. The 2011 report on dietary reference intakes for calcium and vitamin D from the Institute of Medicine: what clinicians need to know. J Clin Endocrinol Metab. 2011;96(1):53-8.

18. Munns CF, et al. Global consensus recommendations on prevention and management of nutritional rickets. J Clin Endocrinol Metab. 2016;101(2):394-415.

19. Holick MF, et al. Evaluation, treatment, and prevention of vitamin D deficiency: an Endocrine Society clinical practice guideline. J Clin Endocrinol Metab. 2011;96(7):1911-30.

20. Lazreg S, et al. Age-related macular degeneration and its risk factors in north Africans living in Algeria and Italy. Ophthalmic Res. 2016;56(3): 145-54.

21. de Koning-Backus APM, et al. Intake of vegetables, fruit, and fish is beneficial for age-related macular degeneration. Am J Ophthalmol. 2019;198:70-9.

22. Agron E, et al. Dietary nutrient intake and progression to late age-related macular degeneration in the age-related eye disease study. Invest Ophthalmol Vis Sci. 2020;61(7):2994-2994.

23. Group A-REDSR. Lutein + zeaxanthin and omega-3 fatty acids for age-related macular degeneration: the Age-Related Eye Disease Study 2 (AREDS2) randomized clinical trial. JAMA 2013;309(19):2005-15.

24. European Food Safety Authority (EFSA). Tolerable upper intake levels for vitamins and minerals. http://www.efsa.europa.eu/sites/default/files/efsa rep/blobserver_assets/ndatolerableuil.pdf. 2006. Accessed 30 April 2020.

25. Ho L, et al. Reducing the genetic risk of age-related macular degeneration with dietary antioxidants, zinc, and omega-3 fatty acids: the Rotterdam study. Arch Ophthalmol. 2011;129(6):758-66.

26. Lançon A, Frazzi R, Latruffe N. Anti-oxidant, antiinflammatory and anti-angiogenic properties of resveratrol in ocular diseases. Molecules. 2016;21(3):304.

27. Cumurcu T, Mendil D, Etikan I. Serum zinc and copper level in age-related macular degeneration. Trace Elem Electrolytes. 2006;23:103-7.

28. Tan JS, et al. Dietary antioxidants and the longterm incidence of age-related macular degeneration: the Blue Mountains Eye Study. Ophthalmology. 2008;115(2):334-41. 
29. Ugarte M, Osborne NN. Recent advances in the understanding of the role of zinc in ocular tissues. Metallomics. 2014;6(2):189-200.

30. Institute of Medicine (US) Panel on Micronutrients. Dietary reference intakes for vitamin A, vitamin K, arsenic, boron, chromium, copper, iodine, iron, manganese, molybdenum, nickel, silicon, vanadium, and zinc. 12 Zinc. Washington (DC): National Academies; 2001. https://www.ncbi.nlm. nih.gov/books/NBK222317/. 2001. Accessed 30 April 2020.

31. Ng AL, et al. Dietary habits, fatty acids and carotenoid levels are associated with neovascular agerelated macular degeneration in Chinese. Nutrients. 2019;11(8):1720.

32. van Leeuwen EM, et al. A new perspective on lipid research in age-related macular degeneration. Prog Retin Eye Res. 2018;67:56-86.

33. Chua B, et al. Dietary fatty acids and the 5-year incidence of age-related maculopathy. Arch Ophthalmol. 2006;124(7):981-6.

34. Souied EH, et al. Oral docosahexaenoic acid in the prevention of exudative age-related macular degeneration: the Nutritional AMD Treatment 2 study. Ophthalmology. 2013;120(8):1619-31.

35. Cho E, et al. Prospective study of dietary fat and the risk of age-related macular degeneration. Am J Clin Nutr. 2001;73(2):209-18.

36. Chiu CJ, et al. Does eating particular diets alter the risk of age-related macular degeneration in users of the Age-Related Eye Disease Study supplements? $\mathrm{Br}$ J Ophthalmol. 2009;93(9):1241-6.

37. Mance TC, et al. The role of omega6 to omega3 ratio in development and progression of age-related macular degeneration. Coll Antropol. 2011;35(Suppl 2):307-10.

38. Seddon JM, George S, Rosner B. Cigarette smoking, fish consumption, omega-3 fatty acid intake, and associations with age-related macular degeneration: the US Twin Study of Age-Related Macular Degeneration. Arch Ophthalmol. 2006;124(7):995-1001.
39. Holick MF. Sunlight, ultraviolet radiation, vitamin $\mathrm{D}$ and skin cancer: how much sunlight do we need? Adv Exp Med Biol. 2014;810:1-16.

40. Noguchi Y, et al. Eldecalcitol for the treatment of osteoporosis. Clin Interv Aging. 2013;8:1313-21.

41. $\mathrm{Wu} \mathrm{W}$, et al. The association between serum vitamin D levels and age-related macular degeneration: a systematic meta-analytic review. Investig Ophthalmol Vis Sci. 2016;57(4):2168-77.

42. Ferreira A, et al. Serum vitamin D and age-related macular degeneration: systematic review and metaanalysis. Surv Ophthalmol. 2020. https://doi.org/ 10.1016/j.survophthal.2020.07.003.

43. Christen WG, et al. Effect of vitamin D and $\omega-3$ fatty acid supplementation on risk of age-related macular degeneration: an ancillary study of the VITAL randomized clinical trial. JAMA Ophthalmol. 2020;138(12):1280-9.

44. Mantell DJ, et al. 1 alpha,25-dihydroxyvitamin D(3) inhibits angiogenesis in vitro and in vivo. Circ Res. 2000;87(3):214-20.

45. Kim KL, Park SP. Association between serum vitamin D deficiency and age-related macular degeneration in Koreans: clinical case-control pilot study. Medicine (Baltimore). 2018;97(33):e11908.

46. Singh A, et al. The association between plasma 25-hydroxyvitamin D and subgroups in age-related macular degeneration: a cross-sectional study. PLoS One. 2013;8(7):e70948.

47. Foss YJ. Vitamin D deficiency is the cause of common obesity. Med Hypotheses. 2009;72(3):314-21.

48. Giovannucci E, et al. 25-hydroxyvitamin D and risk of myocardial infarction in men: a prospective study. Arch Intern Med. 2008;168(11):1174-80.

49. Kheiri B, et al. Vitamin D deficiency and risk of cardiovascular diseases: a narrative review. Clin Hypertens. 2018;24:9.

50. Abdellah MM, et al. Association of serum 25-hydroxyl vitamin D deficiency and age-related cataract: a case-control study. J Ophthalmol. 2019;2019: 9312929. 